\title{
Global Risk Score and Clinical SYNTAX Score as Predictors of Clinical Outcomes of Patients Undergoing Unprotected Left Main Percutaneous Catheter Intervention
}

\author{
Lucky Cuenza ${ }^{\mathrm{a}, \mathrm{b}}$, Marianne P. Collado ${ }^{\mathrm{a}}$, James Ho Khe Sui ${ }^{\mathrm{a}}$
}

\begin{abstract}
Background: Risk stratification is an important component of left main percutaneous catheter intervention (PCI) which has emerged as a feasible alternative to cardiac surgery. We sought to compare the clinical SYNTAX score and the global risk score in predicting outcomes of patients undergoing unprotected left main PCI in our institution.
\end{abstract}

Methods: Clinical, angiographic and procedural characteristics of 92 patients who underwent unprotected left main PCI (mean age $62 \pm$ 12.1 years) were analyzed. Patients were risk stratified into tertiles of high, intermediate and low risk using the global risk score (GRS) and the clinical SYNTAX score (CSS) and were prospectively followed up at 1 year for the occurrence of major adverse cardiovascular events (MACEs), defined as a composite of all cause mortality, cardiac mortality, non-fatal myocardial infarction, stroke, coronary artery bypass, and target vessel revascularization.

Results: There were 26 (28.2\%) who experienced MACEs, of which $10(10.8 \%)$ patients died. Multivariable hazards analysis showed that the GRS (hazard ratio $(\mathrm{HR})=5.5, \mathrm{P}=0.001)$ and $\mathrm{CSS}(\mathrm{HR}=4.3, \mathrm{P}$ $=0.001)$ were both independent predictors of MACEs. Kaplan-Meier analysis showed higher incidence of MACEs with the intermediate and higher risk categories compared to those classified as low risk. Receiver-operator characteristic analysis showed that the GRS has better discriminatory ability than the CSS in the prediction of 1 year MACEs (0.891 vs. $0.743, \mathrm{P}=0.007)$.

Conclusion: The GRS and CSS are predictive of outcomes after left main PCI. The GRS appears to have superior predictive and prognostic utility compared to the CSS. This study emphasizes the importance of combining both anatomic and clinical variables for optimum prognostication and management decisions in left main PCI.

Manuscript submitted October 25, 2017, accepted November 7, 2017

aDepartment of Adult Cardiology, Section of Invasive Cardiology, Philippine Heart Center, East Avenue, Quezon City 1100, Philippines

${ }^{b}$ Corresponding Author: Lucky R. Cuenza, Department of Adult Cardiology, Philippine Heart Center, East Avenue, Quezon City 1100, Philippines.

Email:1rcuenza@gmail.com

doi: https://doi.org/10.14740/cr601w
Keywords: Percutaneous catheter intervention; Global risk score; Clinical SYNTAX score; Left main; Risk stratification

\section{Introduction}

Significant left main coronary artery disease (CAD) is present in $5-7 \%$ of patients undergoing coronary angiography [1]. While coronary artery bypass graft (CABG) surgery has traditionally been the treatment of choice for coronary lesions involving the left main coronary artery, there has been a recent trend regarding the feasibility of left main percutaneous catheter interventions (PCI) in selected patients [2]. As such there is also great interest in the development of risk stratification models to identify whether these patients will have a favorable outlook after the procedure [3]. The importance of risk stratification in these patients is further emphasized when considering the escalating complexity of left main CAD being treated with PCI as well as increasing age and other comorbidities of patients undergoing left main PCI, which may be associated with less favorable clinical outcomes [4].

The SYNTAX score was developed initially evaluating primarily the coronary anatomy to determine the risk of post-procedure adverse events as well as determination of the proper revascularization strategy [5]. Taking into account the absence of clinical factors in its computation, the clinical SYNTAX score (CSS) was then developed and has shown better discriminatory ability than the SYNTAX score alone [6].

It has long been postulated that combining the SYNTAX score with another scoring system will increase its predictive yield [7]. The development of the EuroSCORE, which was initially used to prognosticate post-operative cardiac patients enabled this eventually [8]. Eventually the global risk score (GRS) was developed [9]. It combined the scores of the SYNTAX and the EuroSCORE to better risk stratify patients. Compared to other stand-alone or combined risk scores, the global risk classification seems to be able to discriminate and predict outcomes in patients undergoing unprotected left main PCI and CABG [10]. This study aimed to determine which risk score has better discriminative ability in predicting outcomes in 1 year in patients undergoing left main PCI in our setting. 


\section{Materials and Methods}

This was a prospective cohort study of patients seen at the Philippine Heart Center who underwent left main PCI from 2014 to 2016. The study protocol was approved by our institutional ethics review board. Patients with left main disease who previously have undergone coronary artery bypass or opted for medical management after coronary angiography were excluded from the study. Eligible patients who underwent left main PCI during the study period were enrolled in the study after giving informed consent. The decision to perform PCI was made after initial consultation and discussion with a surgeon and presenting the option of revascularization to the patient. The use of adjunctive therapies, stent types and techniques was left to the discretion of the operator. All patients received second generation drug eluting stents. Patient's risk factors and clinical profiles were determined and the subjects were stratified using both the GRS and the CSS. The SYNTAX score is calculated by a computer program consisting of sequential and interactive self-guided questions [11]. Before undergoing PCI coronary angiograms were reviewed by three interventional cardiologists for determination of the SYNTAX score.

\section{CSS}

The SYNTAX score was combined with a simple clinical risk score incorporating age, ejection fraction, and creatinine clearance to produce the CSS. The CSS derived was then used to stratify patients into three different groups based on their scores: CSS low risk < 30, CSS intermediate risk $30-59$ and CSS high risk $>60$ [12].

\section{GRS}

The additive EuroSCORE was calculated according to the original components [13]. The GRS is a combination of both EuroSCORE and SYNTAX score. The EuroSCORE will stratify three groups to identify different risks (low risk: $0-2$; intermediate risk: 3 - 5; high risk: $>6$ ). The SYNTAX score will stratify into three groups according to tertiles (lowest tertile: $<$ 22; intermediate tertile: 23 - 32; highest tertile: > 33). The GRS system classified patients into three risk groups. The low-risk group will be composed of patients with both low/intermediate EuroSCORE and low/intermediate SYNTAX score. The intermediate-risk group was composed of patients with high EuroSCORE or high SYNTAX score. The high-risk group was composed of patients with both high EuroSCORE and high SYNTAX score [14].

\section{Assessment of clinical outcomes}

The study participants were followed up after procedure and during the hospital stay, via telephone/cell phone calls, use of electronic data and outpatient follow-up. Clinical follow-up
Table 1. Baseline Clinical, Angiographic and Procedural Characteristics

\begin{tabular}{|c|c|}
\hline Characteristic $(\mathrm{n}=92)$ & Total, $\mathbf{n}(\%)$ or \pm SD \\
\hline Age & $62 \pm 12.1$ \\
\hline Sex (male) & $67(72)$ \\
\hline Hypertension & $79(85)$ \\
\hline Diabetes & $35(38)$ \\
\hline Cerebrovascular disease & $7(7.6)$ \\
\hline Chronic kidney disease & $5(5.4)$ \\
\hline Smoking & $43(46)$ \\
\hline Dyslipidemia & $40(43)$ \\
\hline Hx of previous ACS & $22(23.9)$ \\
\hline Hx of previous PCI & $5(5.4)$ \\
\hline Stable angina & $27(30.3)$ \\
\hline Unstable angina/NSTEMI & $32(35.9)$ \\
\hline STEMI & $30(33.7)$ \\
\hline Heart failure & $11(11.9)$ \\
\hline LVEF & $55.8 \pm 10.9$ \\
\hline SYNTAX score & $21.8 \pm 12.2$ \\
\hline EuroSCORE & $2.7 \pm 2.3$ \\
\hline Clinical SYNTAX score & $23.9 \pm 18.5$ \\
\hline Bifurcation lesion & $39(42)$ \\
\hline Ostial & $36(39)$ \\
\hline Thrombus & $10(10.8)$ \\
\hline Number of lesions & $1.9 \pm 0.9$ \\
\hline Lesions treated & $1.4 \pm 0.7$ \\
\hline Number of stents implanted & $1.96 \pm 1.2$ \\
\hline Mean stent length in left main & $23.7 \pm 8.1$ \\
\hline Mean stent diameter & $3.1 \pm 0.7$ \\
\hline Left main only & $36(39)$ \\
\hline Left main plus one vessel & $26(28)$ \\
\hline Left main plus two vessels & $24(26)$ \\
\hline Three vessel with left main involvement & $6(6.5)$ \\
\hline Complete revascularization & $27(29)$ \\
\hline Procedural success & $87(94)$ \\
\hline
\end{tabular}

duration was up to 1 year when the scores were calculated from the date of the index procedure to the end of follow-up date if the patient was free from significant cardiovascular events or mortality; otherwise the follow-up duration was calculated from the index procedure to the date of the incidence of any major adverse cardiovascular events (MACEs). The primary endpoint was any occurrence of MACEs defined as a composite of all-cause mortality, cardiac death, non-fatal myocardial infarction (MI), coronary artery revascularization, new onset of cerebrovascular events, and target vessel revascularization. All-cause mortality includes both cardiovascular (CV) deaths 
and non-CV deaths.

\section{Data analysis}

Quantitative variables were presented as frequency and percentage, and quantitative variables were presented as mean and standard deviation (SD). Variables associated with the hazard (risk) of an event were determined using multivariable Cox proportional hazard analysis. Kaplan-Meier analysis was performed to determine the cumulative incidences of MACEs in 1 year per stratification. Differences between groups were compared using log rank test. The comparisons of discriminatory ability between GRS and the CSS in terms of predicting MACEs were performed with receiver-operator characteristics (ROC) curve analysis. A probability value of $\leq 0.05$ was considered statistically significant. All statistical analyses were performed using STATA SE Version 13 (STATA Corp., TX, USA).

\section{Results}

The different baseline characteristics of the patients are listed in Table 1. The patients' mean age was $62 \pm 12.1$ years, most were male and hypertensive ( $72 \%$ and $85 \%$, respectively), and $23.9 \%$ had a history of a previous acute coronary syndrome (ACS). The mean ejection fraction was $55.8 \pm 10.9$. The mean SYNTAX score was $21.8 \pm 12.2$ and the mean EuroSCORE was $2.7 \pm 2.3$. The rest of the angiographic characteristics are summarized in the table. Three vessel disease with left main involvement was noted in $6.5 \%$ of patients and $29 \%$ underwent complete revascularization. Procedural success was 94\%.

The occurrence of major adverse events is summarized in Table 2. Ten patients died, with seven (7.6\%) of them from cardiovascular causes. Five (5.5) patients had in-hospital death. Thirteen patients $(13 \%)$ had MI, one patient underwent emergency CABG and four (4.3\%) had target vessel revascularization. The distribution of patients based on risk stratification is tabulated in Table 3. On the basis of the CSS patients stratified at low, intermediate, and high risk were $72.8 \%, 20.6 \%$, and $6.5 \%$. The distribution based on the GRS was $59.7 \%$ for the low risk, $28.2 \%$ for intermediate risk, and $11.9 \%$ for high risk, respectively. With regard to the CSS, there were 10 patients each in the low risk and intermediate risk (38.4\% each respectively) and six patients in the high risk group (23\%) who had major adverse events. The cumulative incidences of major ad-
Table 2. Major Adverse Cardiovascular Events at 1 Year (MACE)

\begin{tabular}{ll}
\hline Event $(\mathbf{N}=\mathbf{9 2})$ & $\mathbf{n}(\mathbf{\%})$ \\
\hline Total MACEs & $26(28.2)$ \\
All-cause mortality & $10(10.8)$ \\
Cardiovascular mortality & $7(7.6)$ \\
In-hospital death & $5(5.56)$ \\
MI & $13(13)$ \\
Coronary artery revascularization $(\mathrm{CABG})$ & $1(4.5)$ \\
Stent thrombosis & $2(2.1)$ \\
Target vessel revascularization & $4(4.35)$ \\
Stroke & $3(3)$ \\
\hline
\end{tabular}

verse events were $3.8 \%, 53.8 \%$, and $42.3 \%$ for the GRS low, GRS mid and GRS high, respectively.

The time to event analysis for the occurrence of MACE at 1 year in patients stratified according to each risk score is shown in Figure 1. Patients in the low risk CSS were shown to be $80 \%$ free from adverse events while those in the high risk tertile experienced a $50 \%$ occurrence within 8 months, beyond which only less than $25 \%$ were MACE free. Using the GRS, patients in the low-risk group had a very low likelihood of adverse events at 1 year. Patients in the intermediate risk group showed a $75 \%$ freedom from MACE within 8 months and $50 \%$ in 1 year. Patients in the high risk group of the GRS experienced a 75\% likelihood of major adverse within 5 months with progressive increase in risk for 1 year. Using the log rank test, the significant differences in terms of freedom from MACEs at 1 year between each risk group were evident in both classification systems.

After adjustment for potential confounders, the independent predictors of major adverse events on multivariable analysis included age $(\mathrm{P}=0.006)$, chronic kidney disease $(\mathrm{P}=0.03)$, and a previous history of ACS $(\mathrm{P}=0.04)$. Other significant variables were lower ejection fraction $(\mathrm{P}=0.03)$, higher syntax score $(\mathrm{P} \leq 0.01)$ and the outcome of procedural success $(\mathrm{P}=$ $0.03)$. Both the CSS (hazard ratio $(\mathrm{HR})=4.3, \mathrm{P} \leq 0.01$ ) and the GRS $(\mathrm{HR}=5.5, \mathrm{P} \leq 0.01)$ proved to be important predictors for the occurrence of major adverse events (Fig. 2).

Both risk models showed good discriminative ability. Figure 3 demonstrates the discriminatory ability of the GRS and the CSS in predicting the risk of 1 year MACE. The area under the ROC curve was higher in the GRS than in the CSS $(0.89$

Table 3. Distribution of MACE Based on Risk Stratification

\begin{tabular}{llll}
\hline Scoring system & With MACE, $\mathbf{N}=\mathbf{2 6}(\mathbf{\%})$ & Without MACE, $\mathbf{N}=\mathbf{6 6}(\mathbf{\%})$ & Total, $\mathbf{N}=\mathbf{9 2}$ \\
\hline CSS low & $10(38.4)$ & $57(86)$ & $67(72.8)$ \\
CSS mid & $10(38.4)$ & $9(13.6)$ & $19(20.6)$ \\
CSS high & $6(23)$ & $0(0)$ & $6(6.5)$ \\
GRS low & $1(3.85)$ & $54(81.8)$ & $55(59.7)$ \\
GRS mid & $12(53.8)$ & $12(18.1)$ & $26(28.2)$ \\
GRS high & $11(42.3)$ & $0(0)$ & $11(11.9)$ \\
\hline
\end{tabular}



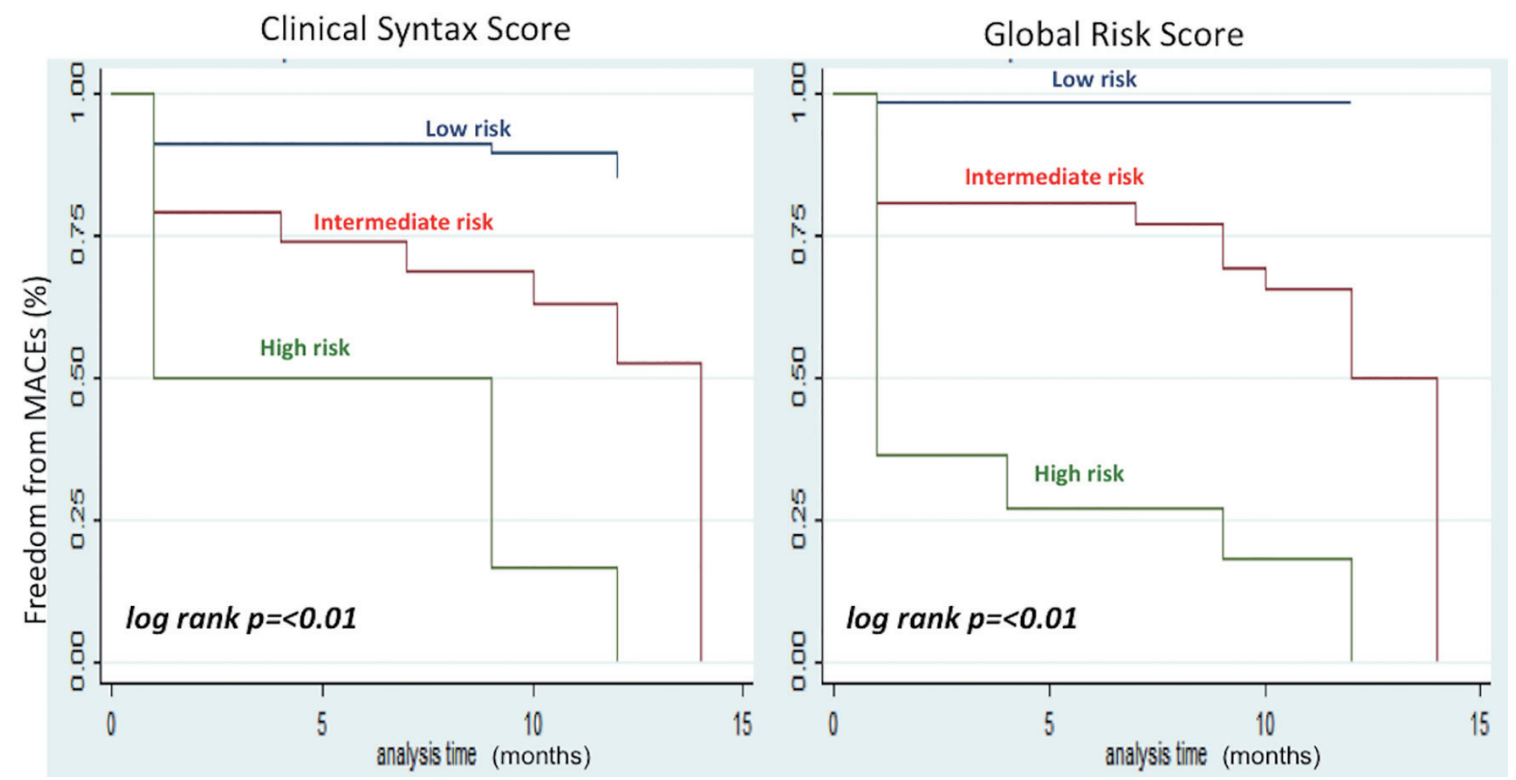

Figure 1. Kaplan-Meier analysis showing the occurrence of MACE at 1 year in patients using the clinical SYNTAX score (left) and the global risk score (right). The occurrence of adverse events was stratified accordingly to low, intermediate and high risk group per scoring system respectively. Differences between groups were compared using log rank test. Analysis time in months.

vs. $0.74, \mathrm{P} \leq 0.01)$.

\section{Discussion}

Risk stratification and the assessment of risk-benefit are two important aspects of clinical medicine [15], and this allows proper appraisal of the patient in both the informed consent and the therapeutic process that will be utilized. Consequently, to enable patients to make the most appropriate informed decision for them as an individual, a suitable method of quantifying risk is essential. In this regard, scoring systems are valuable tools to predict outcomes and help patients and their families to better understand the risks of the procedure to facilitate and be involved of issues relevant to therapeutic strategies.

The results of our study indicate that both the GRS and

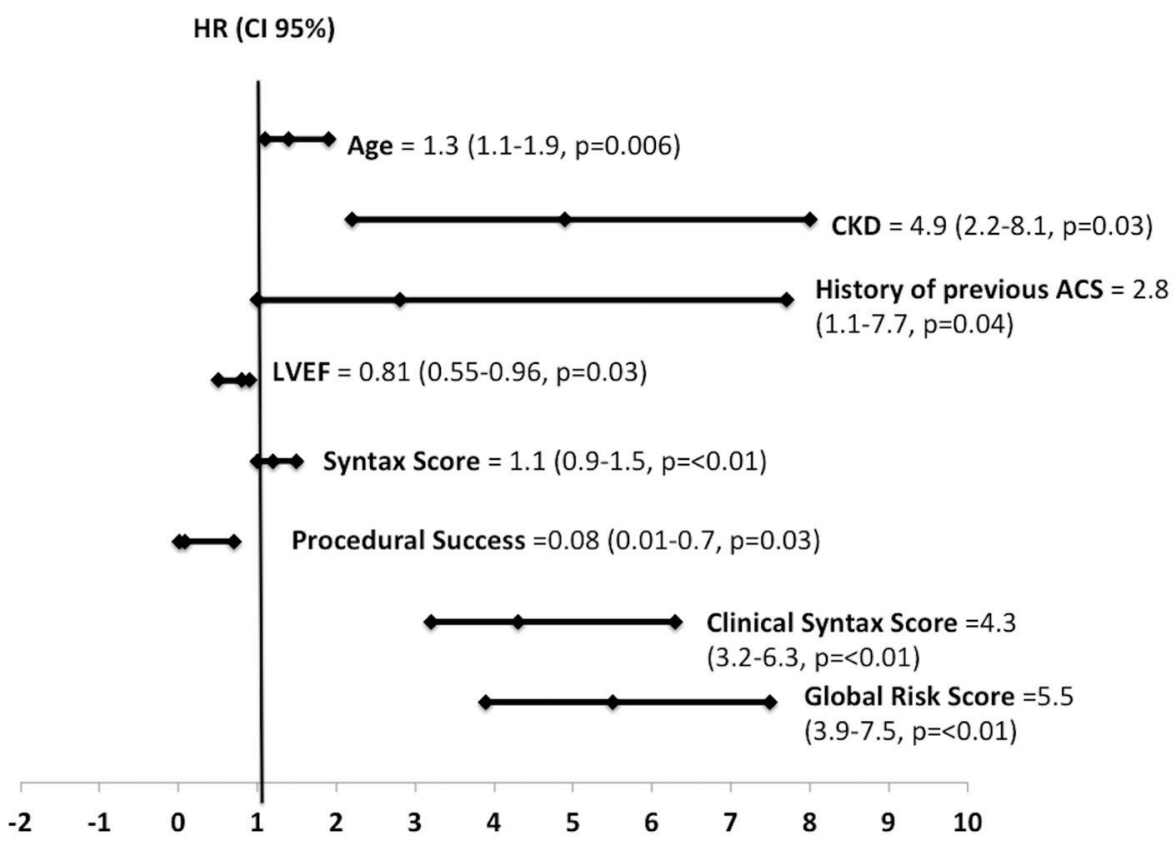

Figure 2. Hazard ratio (HR) plot of statistically significant variables on multivariable Cox proportional analysis. LVEF: left ventricular ejection fraction; ACS: acute coronary syndrome. 


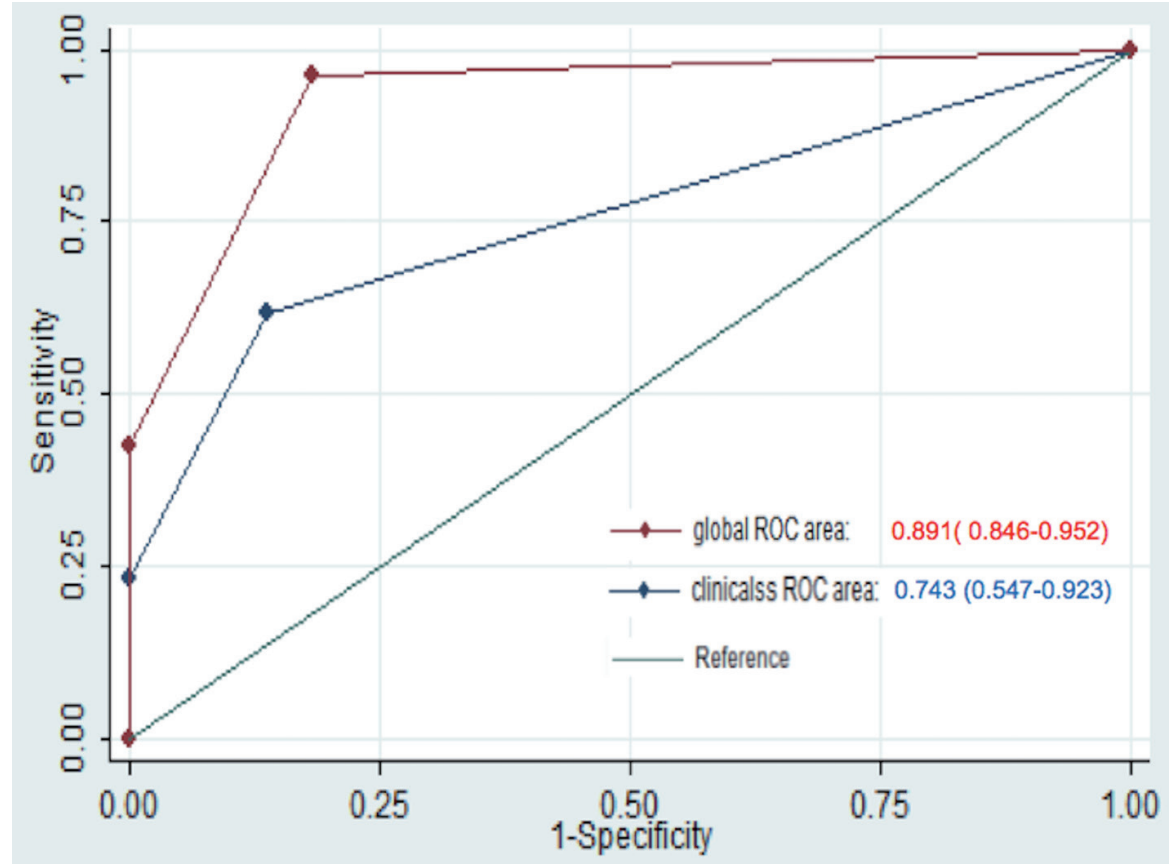

Figure 3. Comparison of area under the curve in predicting major adverse cardiovascular events (MACEs).

the CSS are able to provide prognostic utility in patients undergoing left main PCI. This can be expected as combining clinical data with the angiographic features of the SYNTAX score provide more information than relying on coronary anatomy alone. The global risk score, by combining the angiographic features of the SYNTAX score and the different clinical variables of the EuroSCORE, appears to provide better discriminatory and predictive ability for the occurrence of major adverse events compared to the CSS consistent with the study of Capodanno et al [16]. This is also emphasized in our multivariable hazards analysis as well as in our ROC analysis, where the global risk classification had a significantly higher area under the curve than that of the CSS. A possible explanation for the discrepancy in their discriminatory ability can be due to the fact that the CSS, even after accounting for the angiographic characteristics of the patient and incorporating variables of age, ejection fraction and serum creatinine, is less robust compared to the different variables incorporated in the EuroSCORE. Other variables for predictors of major adverse events in our study include the patient's age, the presence of chronic kidney disease, history of previous ACS, ejection fraction and the SYNTAX score. This is similar to the results of a registry study by Brito et al [17] in that most of the predictive variables in our study were based on the clinical characteristics of the patient. None of the individual angiographic and procedural characteristics (i.e. stent length, thrombus, bifurcation lesion, and ostial lesion) were statistically significant. However, all these anatomic characteristics contribute to the SYNTAX score and in turn may increase the complexity of the CAD, thus contributing to procedural success, which was also considered a significant variable in our study. Even if it was statistically significant, these findings may suggest that the effect of the SYNTAX score alone $(\mathrm{HR}=1.1)$ on our out- comes may be small. A study by Migliorini et al [18] has also suggested that in patients with unprotected left main disease, clinical outcomes of PCI with an everolimus-eluting stent do not appear to be influenced by high anatomical complexity as defined by a SYNTAX score of at least 33. It also provides rationale that the SYNTAX score should be complementary to the patient's clinical features and presentation and should be taken into consideration as a whole in determination of prognosis [19].

Our study is limited by its single center observational nature and small sample size. Studies that examine long term outcomes in patients undergoing left main PCI which compare the use of these two hybrid scoring systems are lacking and thus our results provide additional knowledge regarding the potential prognostic use of these scores. Secondly, both scoring systems were designed to play a role most specially in the risk stratification of higher risk patients. Due to the low sample size, patients stratified in the high risk group may have been underrepresented. It should be noted however that all patients classified under high risk for both the CSS and the GRS experienced major adverse events and the differences in MACEs between different risk profiles reached statistical significance despite the small sample size. For future studies we recommend a larger sample size with possibly equal distribution of all the risk groups for analysis as well as longer follow-up to further validate the use of these scores to clinical practice. Additionally, intracoronary imaging techniques such as intravascular ultrasound (IVUS) were not frequently used to optimize the intervention due to socioeconomic limitations (only two patients had adjunctive IVUS) which may also impact outcomes [20].

The results of our study emphasize the importance of combining both clinical and angiographic characteristics to deter- 
mine appropriate therapy as well as prognosis in this relatively high risk subset of patients. Both scores provide good discriminative and predictive ability for major adverse events in a real world population reflecting current practice in our setting. Risk stratification plays an important part in the decision making process as well as prognostication in patients undergoing left main PCI. It must be noted that no risk prediction model is perfect, with the different nuances involved regarding ease of use, validity and prognostic accuracy. It cannot replace operator skill and good clinical judgment [21]. However, we can use them in our practice as tools of best estimates of risk and as a guide, along with multidisciplinary dialogue involving the Heart Team [22], both to the physician and the patient.

\section{Conclusion}

Both the CSS and the GRS are useful in risk stratification after left main PCI for the prediction of major adverse events. The GRS appears to have better discriminatory ability in predicting outcomes at 1 year compared to the CSS. These risk scores can complement our clinical judgment and decision for optimum management and prognostication.

\section{Acknowledgments}

The authors would like to thank the Philippine Heart Center Section of Invasive Cardiology for their support in providing clinical data for this study.

\section{Competing Interests}

The authors declare that they have no competing interests.

\section{Grant Support}

None.

\section{Financial Disclosure}

None.

\section{References}

1. Seung KB, Park DW, Kim YH, Lee SW, Lee CW, Hong MK, Park SW, et al. Stents versus coronary-artery bypass grafting for left main coronary artery disease. N Engl J Med. 2008;358(17):1781-1792.

2. Park DW, Park SJ. Percutaneous Coronary Intervention of Left Main Disease: Pre- and Post-EXCEL (Evaluation of XIENCE Everolimus Eluting Stent Versus Coronary Artery Bypass Surgery for Effectiveness of Left Main Revascularization) and NOBLE (Nordic-Baltic-British
Left Main Revascularization Study) Era. Circ Cardiovasc Interv. 2017;10(6):e004792.

3. Metzler B, Winkler B. SYNTAX, STS and EuroSCORE - how good are they for risk estimation in atherosclerotic heart disease? Thromb Haemost. 2012;108(6):10651071.

4. Legrand VM, Garg S, Serruys PW, Virtanen KS, Szurawitzki G, Voudris V, Fontanelli A, et al. Influence of age on the clinical outcomes of coronary revascularisation for the treatment of patients with multivessel de novo coronary artery lesions: sirolimus-eluting stent vs. coronary artery bypass surgery and bare metal stent, insight from the multicentre randomised Arterial Revascularisation Therapy Study Part I (ARTS-I) and Part II (ARTS-II). EuroIntervention. 2011;6(7):838-845.

5. Mohr FW, Rastan AJ, Serruys PW, Kappetein AP, Holmes DR, Pomar JL, Westaby S, et al. Complex coronary anatomy in coronary artery bypass graft surgery: impact of complex coronary anatomy in modern bypass surgery? Lessons learned from the SYNTAX trial after two years. J Thorac Cardiovasc Surg. 2011;141(1):130-140.

6. Farooq V, Vergouwe Y, Raber L, Vranckx P, Garcia-Garcia H, Diletti R, Kappetein AP, et al. Combined anatomical and clinical factors for the long-term risk stratification of patients undergoing percutaneous coronary intervention: the Logistic Clinical SYNTAX score. Eur Heart J. 2012;33(24):3098-3104.

7. Serruys P. Integrating lessons from recent clinical trials into practice: Beyond the SYNTAX score. 2010 Angioplasty Summit Transcatheter Therapeutics Asia Pacific, April 29, 2010. Available from from http://www.summittctap.com/2010/AS2010.pdf.

8. Siregar S, Groenwold RH, de Heer F, Bots ML, van der Graaf Y, van Herwerden LA. Performance of the original EuroSCORE. Eur J Cardiothorac Surg. 2012;41(4):746754.

9. Applegate RJ. Toward better stratification of patients with left main disease: value of clinical and angiographic-derived risk scores. JACC Cardiovasc Interv. 2011;4(3):298299.

10. Serruys PW, Farooq V, Vranckx P, Girasis C, Brugaletta S, Garcia-Garcia HM, Holmes DR, Jr., et al. A global risk approach to identify patients with left main or 3-vessel disease who could safely and efficaciously be treated with percutaneous coronary intervention: the SYNTAX Trial at 3 years. JACC Cardiovasc Interv. 2012;5(6):606-617.

11. Palmerini T, Caixeta A, Stone G. A guide to calculating syntax score. Interventional Cardiology. 2012;7(1):2123.

12. Garg S, Sarno G, Garcia-Garcia HM, Girasis C, Wykrzykowska J, Dawkins KD, Serruys PW. A new tool for the risk stratification of patients with complex coronary artery disease: the Clinical SYNTAX Score. Circ Cardiovasc Interv. 2010;3(4):317-326.

13. Roques F, Michel P, Goldstone AR, Nashef SA. The logistic EuroSCORE. Eur Heart J. 2003;24(9):881-882.

14. Sinning JM, Stoffel V, Grube E, Nickenig G, Werner N. The Value of a Combination of SYNTAX score and Euroscore for the prediction of clinical outcomes in patients 
after unprotected left main coronary artery stenting, Paper presented at ESC Congress Paris, 2011.

15. Federspiel JJ, Stearns SC, van Domburg RT, Sheridan BC, Lund JL, Serruys PW. Risk-benefit trade-offs in revascularisation choices. EuroIntervention. 2011;6(8):936-941.

16. Capodanno D, Caggegi A, Miano M, Cincotta G, Dipasqua F, Giacchi G, Capranzano P, et al. Global risk classification and clinical SYNTAX (synergy between percutaneous coronary intervention with TAXUS and cardiac surgery) score in patients undergoing percutaneous or surgical left main revascularization. JACC Cardiovasc Interv. 2011;4(3):287-297.

17. Brito J, Teles R, Araujo P, Sousa JP. Merging syntax score with the Euroscore improves risk stratification of patients undergoing left main angioplasty. The ACROSS registry. Paper presented at ESC Congress, Paris 2011.

18. Migliorini A, Valenti R, Parodi G, Vergara R, Buonamici P, Cerisano G, Carrabba N, et al. Angiographic and Clinical Outcomes After Everolimus-Eluting Stenting for Unprotected Left Main Disease and High Anatomic Coronary Complexity. JACC Cardiovasc Interv. 2016;9(10):1001-1007.
19. Farooq V, Brugaletta S, Serruys PW. Utilizing risk scores in determining the optimal revascularization strategy for complex coronary artery disease. Curr Cardiol Rep. 2011;13(5):415-423.

20. Park SJ, Kim YH, Park DW, Lee SW, Kim WJ, Suh J, Yun SC, et al. Impact of intravascular ultrasound guidance on long-term mortality in stenting for unprotected left main coronary artery stenosis. Circ Cardiovasc Interv. 2009;2(3):167-177.

21. Caggegi A, Capodanno D, Tamburino C. Assessing patients for left main percutaneous coronary intervention considerations and practicalities of risk scores. Interventional Cardiology. 2012;7(1):24-27.

22. Windecker S, Kolh P, Alfonso F, Collet JP, Cremer J, Falk V, Filippatos G, et al. 2014 ESC/EACTS Guidelines on myocardial revascularization: The Task Force on Myocardial Revascularization of the European Society of Cardiology (ESC) and the European Association for CardioThoracic Surgery (EACTS)Developed with the special contribution of the European Association of Percutaneous Cardiovascular Interventions (EAPCI). Eur Heart J. 2014;35(37):2541-2619. 